\title{
crv4, a mouse model for human ataxia associated with kyphoscoliosis caused by an mRNA splicing mutation of the metabotropic glutamate receptor $1(\mathrm{Grml})$
}

\author{
VALERIO CONTI $^{1,2}$, ASADOLLAH AGHAIE ${ }^{3}$, MICHELE CILLI $^{4}$, NATALIA MARTIN ${ }^{3}$, GIANLUCA CARIDI $^{5}$, \\ LUCA MUSANTE $^{2,5}$, GIOVANNI CANDIANO ${ }^{5}$, MAURA CASTAGNA ${ }^{6}$, ALFONSO FAIREN ${ }^{7}$, \\ ROBERTO RAVAZZOLO $^{1,8}$, JEAN-LOUIS GUENET ${ }^{3}$ and ALDAMARIA PULITI ${ }^{1,8}$

\begin{abstract}
${ }^{1}$ Laboratory of Molecular Genetics, and ${ }^{2}$ Renal Child Foundation, G. Gaslini Institute, Genova, Italy; ${ }^{3}$ Unité de Génétique fonctionnelle de la Souris, Institut Pasteur, Paris, France; ${ }^{4}$ Animal Models Facility, Istituto Nazionale per la Ricerca sul Cancro, Genova; ${ }^{5}$ Laboratory on Pathophysiology of Uremia, G. Gaslini Institute, Genova; ${ }^{6}$ Laboratorio di Anatomia Patologica, Department of Surgery, University of Pisa, Pisa, Italy; ${ }^{7}$ Instituto de Neurociencias, CSIC and Universidad Miguel Hernández, San Juan de Alicante, Spain; ${ }^{8}$ Department of Pediatrics and CEBR, University of Genova, Italy
\end{abstract}

Received March 29, 2006; Accepted May 26, 2006

\begin{abstract}
We describe a novel spontaneous autosomal recessive mutation, cervelet-4 (crv4), which arose in a BALB/c strain. Mice homozygous for the mutation exhibit principally a reduced body size, a congenital neurological phenotype characterized by ataxic gait and intention tremor, with no gross anomalies observed in brain or cerebellum, and skeletal anomalies. Using linkage analysis, we mapped the crv4 locus to the proximal region of chromosome 10 , at the location of the Grml gene. Genetic complementation crosses between crv4 and Grml KO mice confirmed that crv4 is a new allele of Grml. Molecular analysis of the Grml gene in mutant mice revealed the insertion of a 190-bp LTR fragment in intron 4. Our results also indicated that the presence of the LTR fragment caused the disruption of the Grml normal splicing process and complete absence of the wild-type protein. crv4 is an interesting model to extend the study of Grml function and the pathological effects of Grml deficiency in vivo.
\end{abstract}

\section{Introduction}

Glutamate is the major excitatory neurotransmitter in the mammalian central nervous system (CNS) and plays an important role in a number of central nervous system functions (1). Metabotropic glutamate receptors (mGluRs), one of the two broad categories in which glutamate receptors are

Correspondence to: Professor Roberto Ravazzolo, Laboratory of Molecular Genetics, G. Gaslini Institute, Largo G. Gaslini 5, 16148 Genova, Italy

E-mail: rravazzo@unige.it

Key words: ataxia, kyphoscoliosis, Grml, retrotransposon insertion, additional exon, splicing error classified, are mainly involved in the regulation of synaptic plasticity, such as long-term potentiation and long-term depression, which are thought to be models of learning and memory (2). mGluRs consist of at least eight subtypes that regulate a variety of intracellular signalling systems via activation of GTP-binding proteins (3). Glutamate receptor metabotropic $1(\mathrm{Grml})$ is present in a number of key CNS structures including the hippocampus, cortex, thalamus and cerebellum and the involvement of this receptor in a variety of disorders including mainly ataxia, epilepsy, ischemia, pain and neurodegenerative diseases is beginning to emerge (1). In general, the biological cause of these disorders can be intrinsic defects of structure or function of cerebellum or its connections (4). An invaluable resource to aid the understanding of the processes underlying these defects are mouse neurological mutations.

In the present study we describe a spontaneous mouse mutation, cervelet-4 (crv4), that occurred in the Grml gene and results from the insertion of a retrotransposon LTR fragment in an intron. This insertion generates abnormal splicing of the gene with occurrence of a premature 'in-frame' stop codon. As a final consequence, the wild-type Grm I protein is not present in crv4/crv4 mutants thus the mutant mouse is a model of Grml-deficiency, the peculiarity of which will be useful to further understand Grml's function and its involvement in human disease.

\section{Materials and methods}

Mice. The crv4 mutation occurred spontaneously in the $\mathrm{BALB} / \mathrm{c} / \mathrm{Pas}$ inbred strain maintained at the Institut Pasteur and was transferred into the Animal Models Facility, Istituto Nazionale per la Ricerca sul Cancro in Genova. The MAI/Pas strain used for mapping of the mutation, is a new inbred from the Institut Pasteur that is derived from wild specimens of the Mus m. musculus subspecies. Grml KO mice were obtained from GlaxoSmithKline (5) and were maintained in the Servicio 
de Experimentación Animal at the Universidad Miguel Hernández of San Juan de Alicante.

Footprint analyses. Footprint analyses were performed according to a previously described protocol (6). In brief, mice were trained to cross an illuminated alley $(5 \mathrm{~cm}$ wide, $80 \mathrm{~cm}$ long) and to go straight to a dark box located at the end of the alley. Their footpads were then coated with a nontoxic, water-based paint, and the floor of the alley was covered with white paper. This test was repeated three times and the footprints were scanned in order to evaluate the deviation of foot placement.

$X$-ray analysis. Mice were briefly narcotized to facilitate radiography, performed by using a LORAD M-IV system. For comparative purposes, identical radiation energy and photographic exposures were used to prepare radiographs of crv4/crv4 and sex- and age-matched control mice.

Genetic mapping. Chromosomal location of the crv4 locus was achieved by genotyping a large set of DNA samples prepared from (BALB/c x MAI)F2 mice, homozygous for the crv4 allele (crv4/crv4), with a collection of microsatellite markers, spaced at $<20 \mathrm{cM}$ and known to be polymorphic between the two parental strains. PCR reactions were carried out using a standard protocol (7) on $100 \mathrm{ng}$ of tail DNA.

Grml genomic PCR. All coding sequences and exon-intron boundaries of the Grml gene were amplified by using primers designed on the sequence of the flanking exons. Exons 2, and 8 and the last exon containing the polyadenylation site, which are 1018, 931 and 1144 bp long respectively, were amplified in 2 overlapping fragments. Primers genlongF (5'-CAATTCC ATAATGCTCATAATGCATCC-3') and genlongR (5'-AAT ACTTTCATGTGTAGACCTTAGTCCAA-3') designed on the intron 4 sequence, were used to specifically amplify the inserted fragment. All PCR amplifications were performed by using the HOT FIREpol DNA polymerase I (Solis Biodyne), with $50^{\circ} \mathrm{C}$ annealing and a final extension of $72^{\circ} \mathrm{C}$ for $10 \mathrm{~min}$. PCR products were analysed on $1 \%$ agarose gel (SeaKem LE agarose, FMC bioproducts) and visualized by classical ethidium bromide staining.

Grml RT-PCR. Total RNA was extracted from normal and mutant cerebella by using Trizol reagent (Gibco) according to the manufacturer's protocol. For RT-PCR reactions, $1 \mu \mathrm{g}$ of total RNA was used to synthetize the first-strand cDNA by using the ImProm-II ${ }^{\mathrm{TM}}$ reverse transcription system (Promega), according to the manufacturer's protocol. The 4237-bp long Grmla isoform was amplified by using the Expand long template PCR system (Roche), with the primers Grm $1 \mathrm{~F} 1$ (5'-AGCCTTGCACCGTCTGAT-3') and GrmlR1 (5'-ACTC CTTGGCATCTCTGG-3'). The Grml cDNA was amplified with the aim of sequencing by using the Expand High Fidelity PCR system (Roche) and the following set of overlapping primers: GrmlF1 (5'-AGCCTTGCACCGTCTGAT-3') and GrmlR2 (5'-CCTGCCAGCAGTACTTTT-3'); GrmlF2 (5'TTCCCAATGATCTTTTTG-3') and GrmlR3 (5'-TGGGAT GTCGAACAGCTG-3'); GrmlF3 (5'-TACCCCCAGGCAG GACTAAG-3') and GrmlR4 (5'-GAGTGAGAACTCTCCC
AC-3'); GrmlF4 (5'-ATGACAGTGCGGGGGTTA-3') and GrmlR5 (5'-TCCAGACACTCAACAAA-3').

Sequencing. Both genomic and RT-PCR products were purified with the ExoSap-IT enzyme (USB) then sequenced on both strands using BIG DYE dideoxy-terminator chemistry (Applied Biosystem) on an ABI 3100 DNA sequencer (Applied Biosystem).

Northern blot analysis. Twenty-five micrograms of total RNA isolated from cerebellum was electrophoresed in a $1.0 \%$ agarose/formaldehyde gel. The gel was blotted onto Hybond-N nylon membrane (Amersham), probed with a 969-bp RT-PCR product (Genbank AF320126, bp 3100 to 4069) of mouse Grml, and reprobed with a 1290-bp RT-PCR product specific for the mouse $\beta$-actin gene (b-act F, 5'-GCATTGTTACCAA CTGGGAC-3'; R, 5'- CCAGAGCAGTAATCTCCTTC-3').

Histological analyses and immunohistochemistry. Animals were deeply anaesthetized with xylazine and ketamine and transcardially perfused with phosphate buffered saline (PBS, $\mathrm{pH} 7.4$ ), followed by $4 \%$ paraformaldehyde (PFA) in $0.1 \mathrm{M}$, $\mathrm{pH}$ 7.4 PBS. Organs were then removed and either embedded in paraffin wax or, after sucrose cryoprotection, frozen in OCT (Tissue Tec, Sakura) for the histological analyses. Sagittal and coronal sections, $5 \mu \mathrm{m}$ each, obtained from paraffin embedded tissues, were stained with Haematoxylin and Eosin or Nissl staining. For immunohistochemistry, both paraffin embedded and frozen brains were sectioned in coronal plane at $5 \mu \mathrm{m}$ (paraffin embedded) or $20 \mu \mathrm{m}$ (frozen) thickness. Sections were pre-incubated $(30 \mathrm{~min})$ in $10 \%$ donkey serum in PBS, rinsed in PBS and incubated overnight at $4^{\circ} \mathrm{C}$ with $5 \mu \mathrm{g} / \mathrm{ml}$ polyclonal anti-Grmla antibody (Upstate Biotechnology). Sections were rinsed in PBS and incubated for $30 \mathrm{~min}$ with the secondary antibody (Donkey anti-rabbit IgG B, Santa Cruz biotechnology). After two rinses in PBS, the sections were processed for $30 \mathrm{~min}$ with the biotin-streptavidin-peroxidase complex (Vectastain ABC Elite kit, Vector) according to the manufacturer's instructions. Sections were then incubated in DAB substrate kit for peroxidase (Vector) and treated with $\mathrm{H}_{2} \mathrm{O}_{2}$ for 1-6 min.

Western blotting. Western blotting was performed according to established protocols $(8,9)$ with some modifications. In brief, cerebella were homogenized in buffer A ( 5 mM Tris pH 7.2, $2 \mathrm{mM}$ EDTA, $10 \mathrm{mM}$ iodoacetamide and protease inhibitors), and then centrifuged at $30,000 \mathrm{x}$ g for $30 \mathrm{~min}$ at $4^{\circ} \mathrm{C}$ to collect a crude membrane pellet. The pellet was re-suspended in buffer B (20 mM Tris- $\mathrm{HCl}$ pH 6.8, $150 \mathrm{mM} \mathrm{NaCl}, 10 \mathrm{mM}$ EDTA, 1 mM EGTA, 1\% Triton X-100, 10 mM iodoacetamide and protease inhibitors mixture). Protein concentration was determined by the Bradford method (Bio-Rad) and samples were separated on $5 \%$ gel by SDS-PAGE. Proteins were electroblotted and protein transfer was monitored by Ponceau Red S staining. Membranes were then incubated with either the anti-Grmla monoclonal antibody (BD Biosciences) at a dilution of 1:2500, or the polyclonal anti-Grmla antibody (Upstate Biotechnology). The Grml bands were detected with an enhanced chemiluminescence system Versa-Doc 4000 (Bio-Rad). 
$\mathbf{A}$
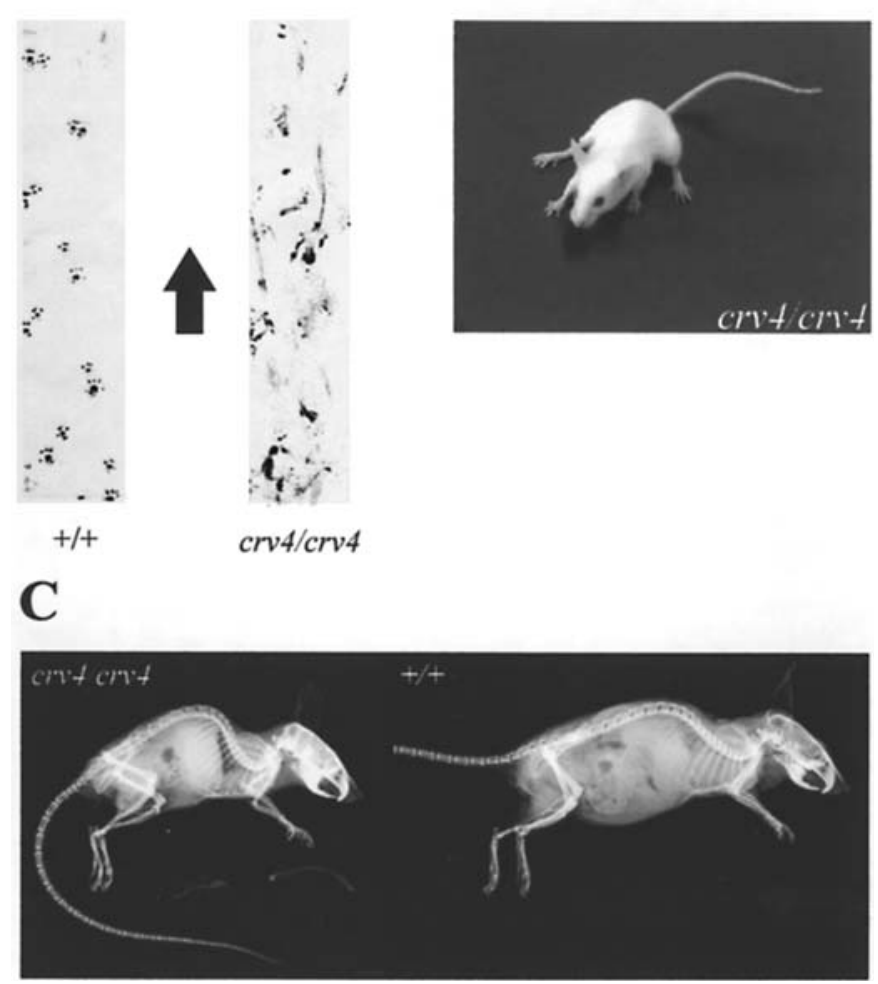

Figure 1. crv4 anomalous gait and posture. (A) Footprint analysis. crv4/crv4 mice walked with a severe wide base rolling motion from side to side and their feet tended to sweep along the floor; as a consequence their gait was severely altered with respect to control mice. (B) $c r v 4 / c r v 4$ mice seemed also to have a mild spasticity of the posterior limbs. (C) X-ray radiography of 10-month-old crv4/crv4 and control (+/+) mice. The crv4/crv4 mouse exhibited thoracic kyphoscoliosis. Due to curvature of the spinal column at the shoulder level, the thoracic region in the crv4/crv4 mouse protruded prominently.

Sequence analysis of the LTR-fragment. The sequence of Grm1 intron 4 , including crv4 genomic insertion, was compared to wild-type sequence by using the BLAST program (http:// www.ncbi.nlm.nih.gov/BLAST/). Open Reading Frame Finder software (http://www.ncbi.nlm.nih.gov/gorf/orfig.cgi) was used to predict the translation of the mutated protein. Alternative Splicing Workbench (http://www.ebi.ac.uk/ asd-srv/wb.cgi) and SpliceView (http://125.itba.mi.cnr.it/ $\sim$ webgene/wwwspliceview.html) programs were used to recognize splicing acceptor and donor sites, polypyrimidine tracts (PPT) and branchpoint (BP) sites in the upstream intronic sequence of the new inserted exon. ESEfinder (http://rulai. cshl.edu/tools/ESE/) and ACESCAN2 (http://genes.mit.edu/ acescan2/) programs were used to evaluate the presence of exonic splicing enhancers (ESE) or exonic splicing silencers (ESS) in the new inserted exon.

\section{Results}

Phenotype of the crv4 mutant. While $+/$ crv4 heterozygous are normal, mice of both sexes homozygous for the new mutation crv4 (crv4/crv4) are viable but exhibit, from birth, a reduction in size by roughly $1 / 3$. Homozygous females show very reduced fertility while males seem to be sterile. Anomalous posture, whole body tremor during movement and ataxic gait
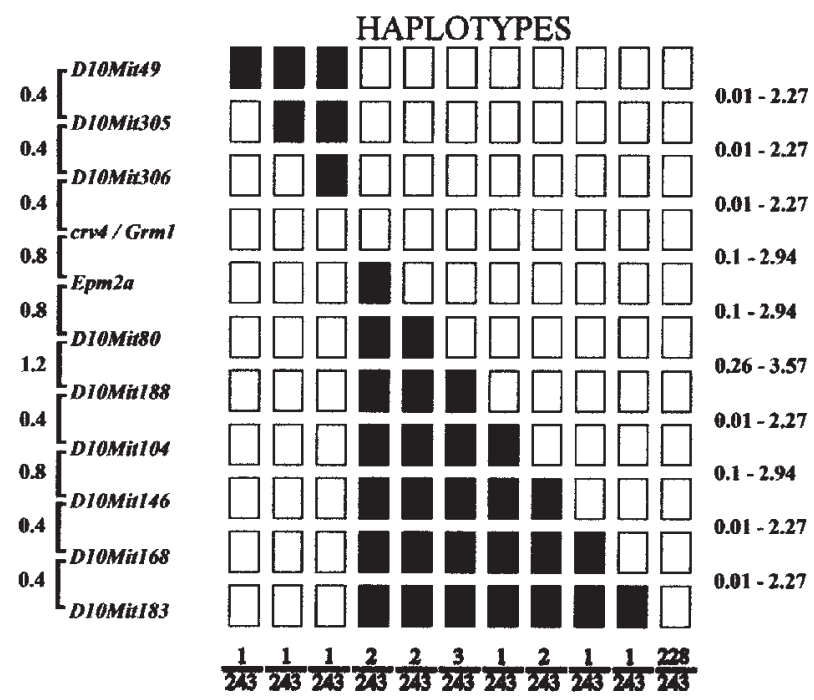

Figure 2. Haplotypes diagram of markers used for mapping the crv4 locus Distribution of the different haplotypes among 121 offspring of an intercross between strain BALB/c, where the mutant allele appeared, and strain $\mathrm{MAI} / \mathrm{Pas}$. All mice but one in this diagram were homozygous for the mutant allele $c r v 4$ while one unaffected mouse progeny, tested as $+/ c r v 4$, was added (243 meiosis in total). Filled rectangles, MAI/Pas alleles; white rectangles, $\mathrm{BALB} / \mathrm{c}$ alleles. The molecular markers used for genotyping are on the left. The intervals between any two markers are provided with confidence intervals at the $5 \%$ risk level. Genotyping of these haplotypes indicates that the locus for crv4 is within a $1.2-\mathrm{cM}$ interval, flanked by markers D10Mit306 and Epm2a, which is equivalent to $2 \mathrm{Mbp}$ of mouse DNA. Marker order and distances established from our cross are in perfect fit with the sequence data available.

are the main features which became clearly evident when the mice started walking. Footprint analysis indicated that mutant mice walked with a severe wide base rolling motion from side to side, they could not walk along a straight line and their feet tended to sweep along the floor. The impairment of motor coordination in crv4/crv 4 mice also seemed to be associated to a mild spasticity of the posterior limbs (Fig. 1A and B). The morphology of the cerebellum appeared unaffected by the crv4 mutation, as shown in hematoxylin and eosinand Nissl-stained material. Purkinje cell number, size and distribution were also normal. No gross anomalies were found in the hippocampus or elsewhere. crv4/crv4 mice exhibited an ocular anomaly, either unilateral or bilateral, with their eyes being more closed than in the control. Eye section revealed no gross anomalies. Twelve crv4/crv 4 mice ( 6 females and 6 males), 6/8 months old and BALB/c controls were sacrificed and investigated for the presence of additional phenotype anomalies. The following features were frequently observed: an increased amount of interscapular brown fat, which was nearly doubled in females compared with control mice; and a kyphoscoliotic defect, particularly evident in females, upon necropsy analysis and by alizarin red/alcian blue skeletal staining and X-ray analysis in three additional affected animals (Fig. 1C).

Genetic mapping of the mouse crv4 locus. Genetic mapping was performed in two steps on a total sample of $121 \mathrm{crv} 4 / \mathrm{crv} 4$ F2 mice. An initial chromosomal assignment using 21 DNA samples of affected animals suggested linkage of the crv4 locus with several markers of chromosome 10. High resolution 


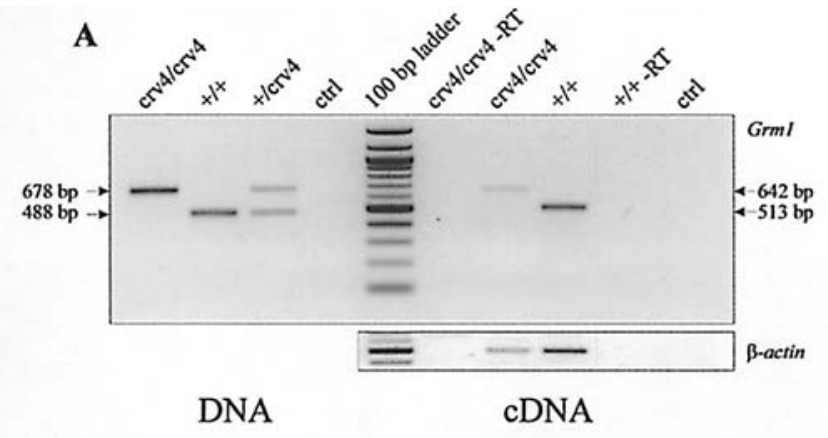

B

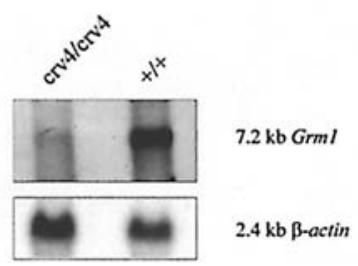

Figure 3. (A) Genomic and RT-PCR analysis of crv4 mutants. In both cases the upper bands represent the inserted sequence, the lower bands represent the wild-type sequence. cDNA insertion is $139 \mathrm{bp}$ long, while the entire insertion is $190 \mathrm{bp}$ long at genomic level. (B) Northern blot analysis of Grm1 expression in wild-type and crv4/crv4 mice. In crv4/crv4 mice, the Grm1 main transcript is detectable at very low levels. As a control for RNA quantity, the same blot was reprobed with a $B$-actin cDNA fragment.

mapping in the critical region with all 121 samples indicated that the crv4 locus maps within the 1.2-cM interval flanked by markers D10Mit306 and Epm2a (Fig. 2). In this interval, the Grml gene appeared as a strong candidate because of striking similarities between the pathophysiological features reported for the Grml knockout $\left(\mathrm{Grml}^{-/}\right)$and our crv4 mutant allele $(5,10,11)$.

crv4 is a new allele at the Grm1 locus. Heterozygous $+/$ crv4 mice were crossed with $\mathrm{Grml}^{+-}$mice, in which one copy of the Grml gene was disrupted by the insertion of a $l a c \mathrm{Z} /$ neo $^{\mathrm{r}}$ expression unit in the domain encoding the second intracellular loop of the seven transmembrane domain of the molecule (5). Both crv4 and Grml are null mutations. As expected for recessive mutations, on the average $1 / 4$ of the $F_{1}$ progeny obtained from this cross exhibited the neurological phenotype characteristic of crv4, confirming that crv4 is indeed a new null allele at the Grml locus.

Molecular characterization of the crv4 mutation. To assess whether crv4 mice have any structural change in the coding sequence of the Grml gene, we checked the sequence of all known exons of the Grml gene in crv4/crv4 mutant mice as well as the sequence of the regions flanking the splicing sites by amplifying genomic DNA fragments encompassing the whole region. We found no mutation. We then decided to analyse the Grml cDNA derived from the cerebellum of the crv4/crv4 mutant mice and control. We observed an amplification product of the expected size, corresponding to the Grmla isoform (12) in the control mice, but the unique product amplified from crv4/crv4 mice cerebellum cDNA showed an increased size leading us to suspect that an insertion might have occurred in the genomic sequence leading to abnormal splicing. To test this hypothesis and identify the suspected insertion site at the RNA level, a series of overlapping RT-PCR followed by cDNA sequencing were performed and a 139-bp insertion between exons 4 and 5 was found corresponding to an inserted DNA fragment of $190 \mathrm{bp}$ at the genomic level (Fig. 3). BLAST analysis indicated that the inserted segment shares $94 \%$ homology with 5' LTR and primer binding sequences of the Mus $m$. domesticus endogenous retroviral element MuERV-L (Y12713) but lacks the other specific MuERV-L features. The LTR fragment was inserted in the same orientation as the Grml gene, and interferes with the normal splicing of the gene by inducing the transcription of an additional exon composed of $23 \mathrm{bp}$ from intron 4 and $116 \mathrm{bp}$ from the LTR-fragment, with an in-frame stop codon (Fig. 4A). Since no evidence of wild-type mRNA presence was obtained by RT-PCR, with either full-length cDNA or with exon 4 and 5 specific primers at different PCR conditions, we assumed that the only transcribed mRNA was the mutated one and, as a consequence, that a wild-type protein was not translated from the mutant transcript. Northern blot analysis of RNA from wild-type and mutant mice (Fig. 3) demonstrated the presence of a Grml specific band although at very low levels.

We investigated the $500 \mathrm{bp}$ intronic sequence encompassing the insertion site for the presence of specific features, which could have favoured the insertion and the preferential expression of the mutant spliced mRNA. The insertion interrupts a 72-bp long SINE sequence of the ID4 family (MUSID4) causing a rearrangement of 18 SINE specific bp (Fig. 4B).

Furthermore, the inserted LTR-fragment sequence introduces a donor 'GT' site, at least 7 murine exonic splicing enhancers (ESEs) and a sequence homologous to a human known silencer (Fig. 4B). An acceptor splice site, polypyrimidine tracts (PPTs) and a branchpoint site, necessary for correct splicing, were identified in a range of $34 \mathrm{bp}$ upstream of the new exon.

Expression of Grm1 protein in crv4 mice. Western blot and immunohistochemistry analyses showed absence of the wild-type Grml protein in the cerebellum, consistent with the absence of wild-type mRNA. Western blot and immunohistochemistry analyses were performed by using antibodies that recognize the Grmla isoform. In Western blotting, monoclonal and polyclonal anti-Grm1 antibodies recognized an $\sim 140-\mathrm{kDa}$ band corresponding to receptor monomers, and a higher molecular weight band, which may correspond to receptor dimers $(9,13)$, in control mice, whereas no protein expression was found in cerebellar extract from crv4/crv4 mice (Fig. 5A). In the cerebellum, Grml is expressed by Purkinje cells perisinaptically with respect to parallel fiberPurkinje cell synapses. Golgi, basket, stellate and Lugaro cells also express Grml (14). Immunohistochemistry performed on frozen and paraffin embedded cerebellar sections with a polyclonal antibody raised against the C-terminal region of Grmla demonstrated the absence of wild-type Grml protein in all layers of the cerebellum (Fig. 5B).

\section{Discussion}

In this paper we report the genetic and phenotypic analysis of cervelet-4 (crv4), a new spontaneous mouse model that exhibits 


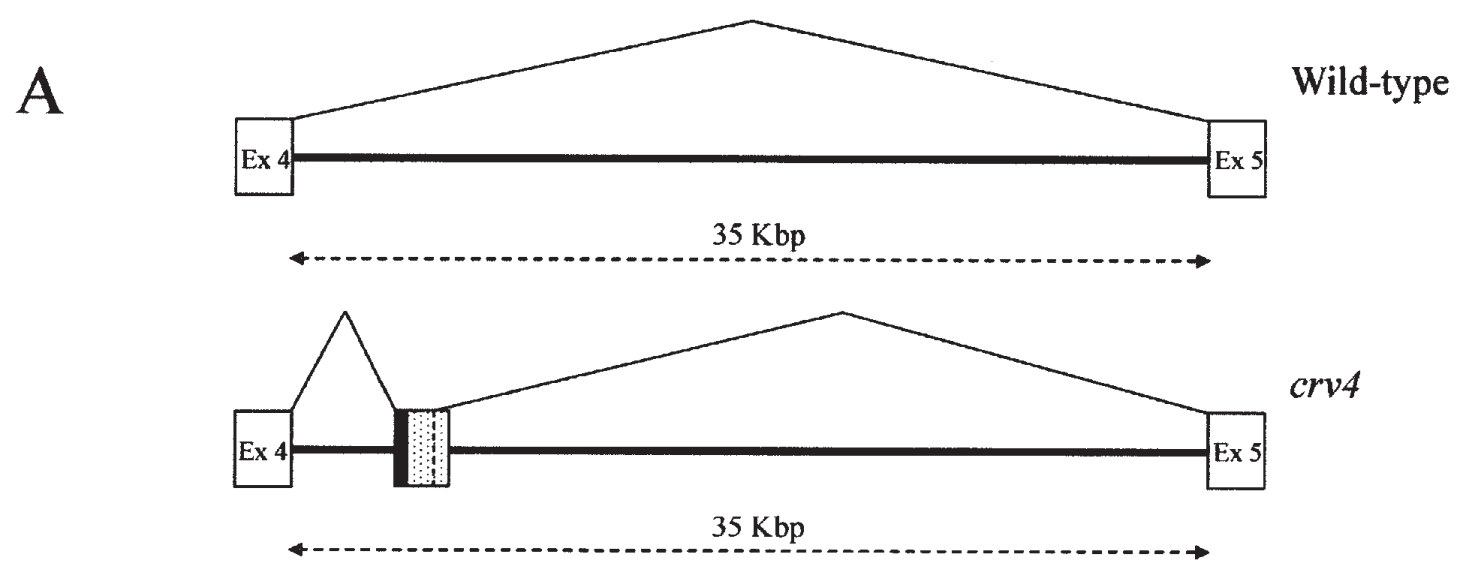

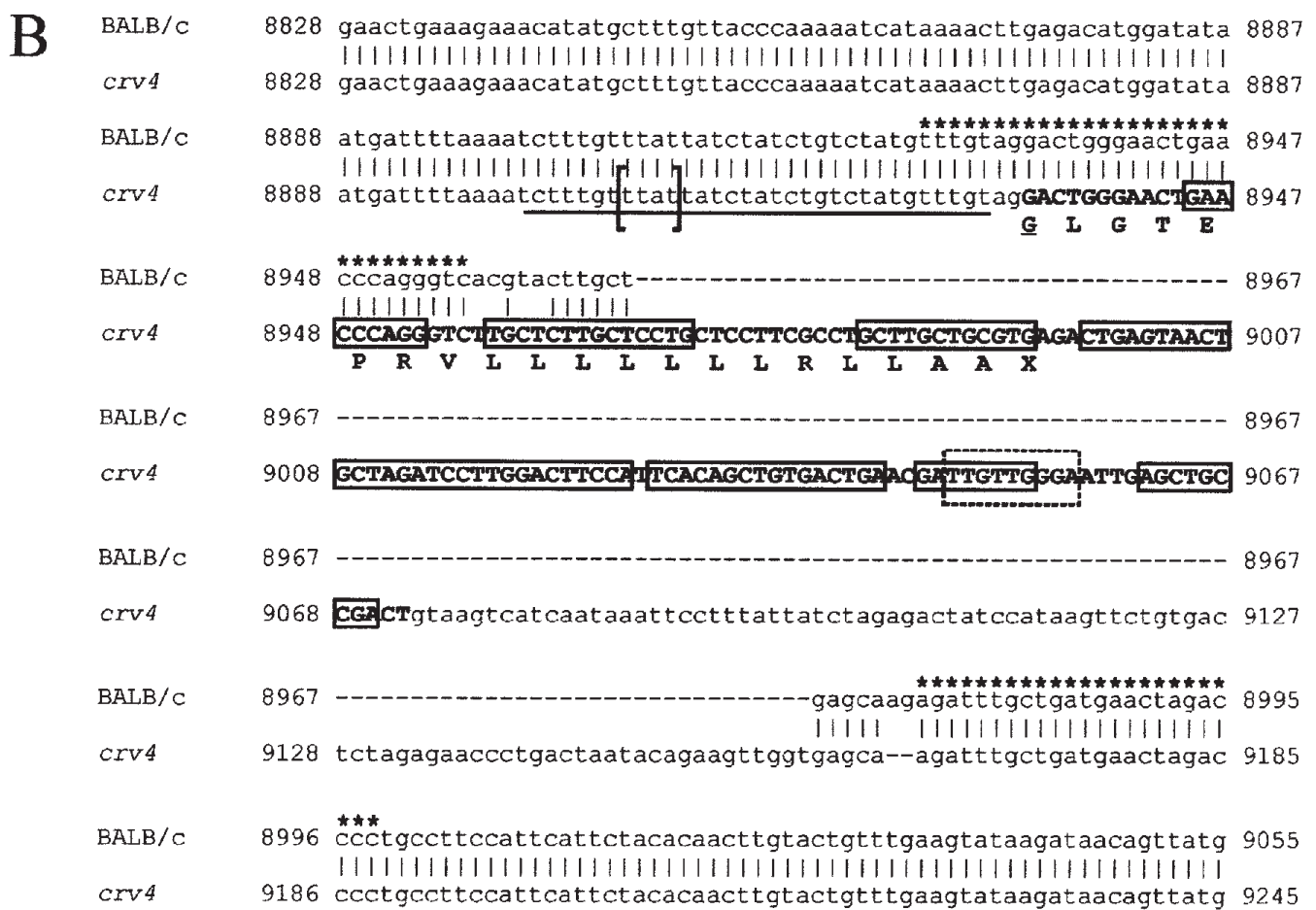

Figure 4. Sequence analysis of Grm1 intron 4. (A) Genomic structure in-between exons 4 and 5 of wild-type and crv 4 mice. Wild-type and possible aberrant splicing mechanisms are indicated as thin lines. Exons 4 and 5 are also indicated. Dotted box, the LTR fragment insertion, the spliced part of the LTR is delimited by the vertical outlined line; black box, the part of intron 4 that is spliced in crv4. (B) Nucleotide sequence comparison of wild-type and $c r v 4$ mice. The alignment between wild-type and crv4 sequences is indicated. Uppercase bold letters represent the new inserted exon, the relative translation is also indicated. The polypyrimidine tract is underlined, and the branchpoint tract is indicated by squared parenthesis. Open boxes, ESE sequences; outlined box, the ESS sequence; stars, the position of SINE specific regions.

a recessive phenotype, characterized mostly by congenital ataxia and intention tremor. This ataxic mouse, in which the Grml gene carries an insertion affecting the normal splicing process with consequent loss of the wild-type protein, is of particular interest both as a model for the identification of molecular mechanisms of ataxia pathogenesis and because of the peculiarity and originality of the mutation mechanism.

The crv 4 mutation is the consequence of the insertion of a 190-bp retrotransposon LTR fragment, homologous to the MuERV-L element, which occurred in intron 4 of the Grml gene. The insertion caused the introduction of an extra exon and an in-frame stop codon in the Grml mRNA sequence. Since no wild-type Grml RNA was detected by RT-PCR, we concluded that the additional exon is constitutively spliced in the mature mRNA while the normal splicing is disrupted. Integration of retroviruses or retrotransposons into the host chromosome have already been reported to result in the inhibition or activation of the expression of targeted genes. The mouse mutations dilute (Myo5a - Chr 9) (15) and hairless ( $\mathrm{Hr}$ - Chr 14) (16) are examples of such spontaneous mutations caused by retroviral insertion. In these mutants, the insertion, which occurred in introns, was associated to a reduced or aberrant expression of the endogenous gene leading to dilute and hairless phenotypes respectively. Interestingly, in these models the proviral excision through homologous recombination between the two Long Terminal Repeats (LTR), leaving a 'solo LTR' in the host chromosome, was associated to a reversion of the phenotype to normal, thus indicating that 


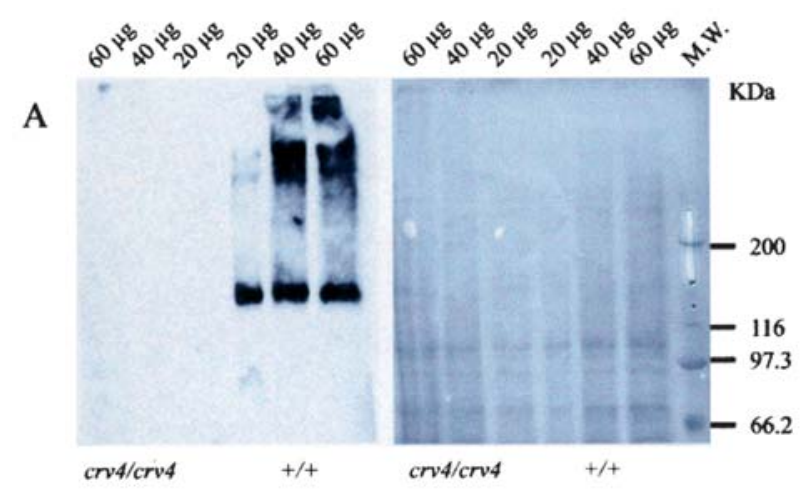

B

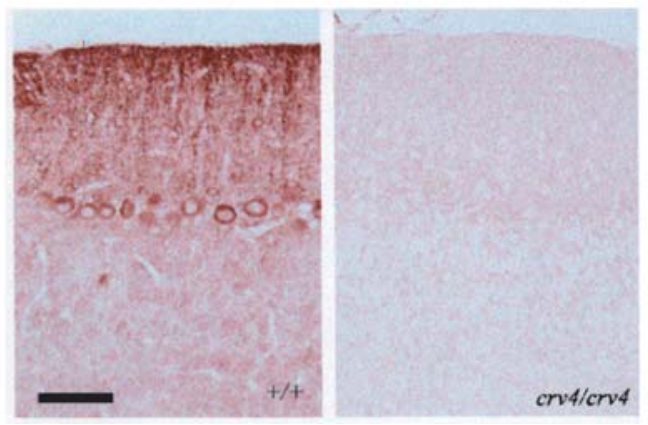

Figure 5. Grml expression in wild-type and crv4 mice cerebella. (A) Western blot analyses performed with antibodies that recognise the Cterminal domain of $\mathrm{Grm} 1$ protein indicate no Grm1 expression in cerebellar membrane extracts from crv4/crv4 mice. (B) No staining was observed in any cerebellar structure of $\operatorname{crv} 4 / \mathrm{crv} 4$ mice by immunohistochemistry, compared to normal control mice. Bar, $50 \mu \mathrm{m}$.

a 'solo LTR' has no mutagenic effect. In the case of the dilute and hairless genes, the presence of the intronic 'solo LTR' allowed the expression not only of an aberrant, but also a normal mRNA form, sufficient to revert the anomalous phenotype (17); this is in agreement with the well-known mechanism by which homologous recombination between two LTRs has led to the production of 'solo LTRs' that are scattered randomly throughout the genome with no consequence on phenotype (18). To the best of our knowledge, only one case in which the insertion of a 'solo LTR' in an intronic region was associated with a pathological phenotype has been reported so far (19). In that case, the insertion of a 520-bp 'solo LTR' (conserving all the classical LTR features) in intron 22 of the $m d r-3$ gene caused disruption of the normal splicing process. In all other reported cases, the presence of intronic 'solo LTRs' seems to be associated to normal phenotype (19). Thus, the crv4 inserted sequence is the smallest LTR-fragment reported to be inserted in an intronic region and to be associated with an aberrant phenotype in mice. To elucidate why the crv4 LTR-fragment, which is only $190 \mathrm{bp}$ long, is able to induce an aberrant splicing of the Grml gene, we analysed the inserted sequence and its flanking intronic regions with softwares that can recognize splicing regulation sequences. Accurate and efficient pre-mRNA splicing is essential to ensure correct gene expression and specific consensus sequences (5' acceptor splice site, branch site and $3^{\prime}$ donor splice site) are found at virtually all exon-intron joints (20). Interestingly, acceptor splicing sites were found in the analysed region in both crv4 and wild-type mice. On the contrary, no donor splice sites were identified in approximately $500 \mathrm{bp}$ encompassing the intronic site of insertion in the wild type, while several donor splice sites were predicted in the mutant sequence, among which is the effective crv4 spliced donor site. In addition, branch point (BP) and Polypyrimidine tracts (PPTs) were also found in the first $34 \mathrm{bp}$ flanking the acceptor site, a distance compatible with that present in normal spliced exons (21). Additional regulatory cis-elements are exonic splicing enhancers (ESEs) and exonic splicing silencers (ESSs). These sequences are known to regulate splicing positively (ESEs) or negatively (ESSs) by the stimulation or inhibition of splicing machinery recruitment $(22,23)$. ESEs, in particular, may be present in most, if not all, exons including constitutive ones (exons always included in the mature mRNA, even in different mRNA isoforms) (24). In the new exon generated in crv4 mRNA, several putative ESEs and only one ESS sequence, in part overlapping one of the predicted ESEs, were predicted (Fig. 4B). It is known that ESEs, when present together, act in a dominant manner over adjacent ESSs (25). Consistent with the absence of detectable wild-type Grm1 mRNA in crv 4 mutants, and because of the high ratio between the number of predicted ESEs and ESSs, we hypothesised that the new additional exon was spliced in the mRNA as a constitutive one. In order to evidence the presence of specific features, such as repeats containing sequences, which could have favoured the insertion of the identified fragment, the intron 4 sequence of the Grml gene was analyzed. The results showed that the LTR-fragment is localized in the central region of a SINE element. SINEs are small elements, usually $100-400 \mathrm{bp}$ in length, which lack a protein-coding sequence and therefore rely on other elements for their retrotransposition (18). It has been reported that human Alus, and B1 and B2 rodent SINE sequences can mediate the integration of flanked sequences by homologous or illegitimate recombination $(26,27)$. In addition, it has been reported that LTR containing retrotransposons can contain 'hot spots' for SINE insertion (28). Consistent with these observations, we hypothesise that such an insertion could have been mediated by a two-step process in which both the SINE and the LTR retrotransposon may have played a role. Overall, the peculiarity of the crv4 mutation underlines the importance of mutations leading to gene function disruption through mechanisms different from simple variations in coding sequences and involving splicing regulatory sequences located far from coding regions.

Expression of the Grml mRNA was investigated by two techniques. Northern blot analysis detected in crv4/crv4 mice a Grml specific band at a very low level. The size of the detected band appeared to be approximately the same as in the control, which was expected since the difference between the wild type and the mutated form consists only in $139 \mathrm{bp}$, a difference not detectable by Northern blot analysis. Thus, by Northern blot analysis we were not able to determine if the observed band resulted from an aberrant or from a residual normal Grml RNA splicing process. On the contrary RT-PCR, which could discriminate the two differently spliced forms, indicated the presence of the mutation as a unique expressed mRNA form. The Northern blot and RT-PCR results together indicate that only the mutant form was present in crv4 homozygous mice although at a very low level. It is noteworthy that mRNAs containing premature-termination codons as a 
result of mutations are hampered to be translated in truncated and potentially harmful proteins by a cell surveillance pathway known as nonsense-mediated mRNA decay (NMD) (29). It is thus possible that the low level of mRNA detected in affected mice, reflecting its very low stability, could result from an NMD mechanism. In case some mutated mRNA molecules escape the surveillance system, a short polypeptide could be produced.

The predicted crv4 protein, shorter than the wild type (415 aminoacids instead of 1,199) retains 396 aa at the N-terminal to which 19 aa derived from the intronic and inserted LTRfragment are added (Fig. 4B). Since the seven transmembrane domains of the Grml protein start from aminoacid 594 (2), we hypothesise that the mutated protein loses its membrane anchoring and, if not degraded, can reach the plasmatic membrane and be secreted. Previous reports provide some support to the hypothesis that naturally secreted isoforms of the Grml protein exist. A short form of Grml mRNA (E55) was detected by RT-PCR in mice although the presence of its translated product was not reported. The predicted protein was 321 aa long, containing only the extracellular domain, which could possibly be secreted (30). In addition, Selkirk and collaborators obtained in vitro the synthesis of a secreted polypeptide by transfecting a Chinese hamster ovary cell line with a construct containing residues 1-592 of the human Grm1 $\mathrm{N}$-terminal domain. They demonstrated that this secreted protein maintains a pharmacological profile similar to that of the native receptor and the ability to bind glutamate (31).

Unfortunately, the unavailability of Grml antibodies specific for the N-terminal part of the protein makes the detection of the crv4 truncated protein difficult to achieve. It is worth noting that, if the truncated protein was secreted, it could have great implications in the extracellular glutamate balance, probably influencing the interactions of glutamate with other receptors. The potential presence of a secreted truncated protein represents a unique feature of crv 4 mice, compared with the previously described Grml null mice $(5,10,11)$. This could be the reason why additional anomalies, such as kyphoscoliosis, which have not been described in Grml null mice $(5,10,11)$, have been observed in crv4/crv4 mice. On the contrary, the eye defect observed in crv4/crv4 mice may be a consequence of the impairment of the conditioned eyeblink response which was already observed in the Grml KO mice by electrophysiological tests (11). Recently, a new mouse mutant carrying a null mutation of the Grml gene in a 129P2/ OlaHsd-C57BL/6 genetic background has been generated (Grm $1^{\text {tmlDgen }}$, direct data submission to Mouse Genome Database (MGD), Deltagen and Lexicon Knockout Mice section, MGI, The Jackson Laboratory, Bar Harbor, Maine; http://www.informatics.jax.org/external/ko/). Interestingly, the main features associated to the neurologic phenotype and reported in Grml tmlDgen homozygous animals are decreased body weight and length, and bone mineral density and content in mice at 49 days of age. The bone density defect reported in homozygous Grm $1^{\text {tmlDgen }}$ mice and the skeleton anomaly observed in crv4/crv4 mice could represent the effects of a unique molecular mechanism. Variable expressivity of the bone phenotype between the two mouse mutants may be due to the different genetic backgrounds of the two animals. Both crv4 and Grm1 $1^{\text {tmIDgen }}$ mouse skeletal observations could be related to recent evidence supporting a role of glutamate signalling in bone remodelling (32). Whilst functional glutamate signalling has been demonstrated in osteoblasts and osteoclasts in vitro, the effects of modulation of glutamate signalling in vivo are still unclear. The crv4 mouse skeletal anomaly may be considered the first in vivo evidence that an impairment of glutamate pathway may be associated to a bone anomaly.

Functional clues of the pathophysiological processes involved in the crv 4 mouse phenotype anomalies could have a great significance in evaluating in vivo new and unknown functions of the Grml gene in tissues different from nervous tissues.

Although no Grml mutations have been reported so far as responsible for ataxia in hereditary forms of the disease, evidence indicates that the lack of a normal Grml protein function because of auto-antibody production may be associated to ataxia in patients affected by Hodgkin's disease (33). Other evidence coming from the study of transgenic mouse models of SCA1 disorder (34) demonstrates the role of glutamate signalling as central to the normal functioning of cerebellum and indicates that alterations in glutamate signalling may play an important role in the pathophysiology of these neurological disorders. For all of these reasons, the study of crv4 mice should help to unravel the pathogenetic mechanisms underlining neurological and associated phenotype anomalies.

\section{Acknowledgements}

We would like to thank our colleague Dominique SimonChazottes, Unité de Génétique fonctionnelle de la Souris, Institut Pasteur, Paris, for her kind help in the genetic mapping data analysis. This work was funded in part by a grant from the Italian Ministry of University (FIRB project) to R.R.

\section{References}

1. Bordi F and Ugolini A: Group I metabotropic glutamate receptors: implications for brain diseases. Prog Neurobiol 59: 55-79, 1999.

2. Pin JP and Duvoisin R: The metabotropic glutamate receptors: structure and functions. Neuropharmacology 34: 1-26, 1995.

3. Aronica E, Catania MV, Geurts J, Yankaya B and Troost D: Immunohistochemical localization of group I and II metabotropic glutamate receptors in control and amyotrophic lateral sclerosis human spinal cord: upregulation in reactive astrocytes. Neuroscience 105: 509-520, 2001.

4. Klockgether T and Evert B: Genes involved in hereditary ataxias. Trends Neurosci 21: 413-418, 1998.

5. Conquet F, Bashir ZI, Davies CH, et al: Motor deficit and impairment of synaptic plasticity in mice lacking mGluR1. Nature 372: 237-243, 1994.

6. Norreel JC, Jamon M, Riviere G, Passage E, Fontes M and Clarac F: Behavioural profiling of a murine Charcot-MarieTooth disease type 1A model. Eur J Neurosci 13: 1625-1634, 2001.

7. Jaubert J, Jaubert F, Martin N, et al: Three new allelic mouse mutations that cause skeletal overgrowth involve the natriuretic peptide receptor $\mathrm{C}$ gene (Npr3). Proc Natl Acad Sci USA 96: 10278-10283, 1999.

8. Ray K, Fan GF, Goldsmith PK and Spiegel AM: The carboxyl terminus of the human calcium receptor. Requirements for cellsurface expression and signal transduction. J Biol Chem 272: 31355-31361, 1997.

9. Ray K and Hauschild BC: Cys-140 is critical for metabotropic glutamate receptor-1 dimerization. J Biol Chem 275: 34245-34251, 2000. 
10. Aiba A, Chen C, Herrup K, Rosenmund C, Stevens CF and Tonegawa S: Reduced hippocampal long-term potentiation and context-specific deficit in associative learning in mGluR1 mutant mice. Cell 79: 365-375, 1994.

11. Aiba A, Kano M, Chen C, et al: Deficient cerebellar long-term depression and impaired motor learning in mGluR1 mutant mice. Cell 79: 377-388, 1994.

12. Simonyi A, Xia J, Igbavboa U, Wood WG and Sun GY: Age differences in the expression of metabotropic glutamate receptor 1 and inositol 1,4,5-trisphosphate receptor in mouse cerebellum. Neurosci Lett 244: 29-32, 1998

13. Cinque C, Zuena AR, Casolini P, et al: Reduced activity of hippocampal group-I metabotropic glutamate receptors in learning-prone rats. Neuroscience 122: 277-284, 2003.

14. Knopfel T and Grandes P: Metabotropic glutamate receptors in the cerebellum with a focus on their function in Purkinje cells. Cerebellum 1: 19-26, 2002.

15. Copeland NG, Hutchison KW and Jenkins NA: Excision of the DBA ecotropic provirus in dilute coat-color revertants of mice occurs by homologous recombination involving the viral LTRs. Cell 33: 379-387, 1983.

16. Stoye JP, Fenner S, Greenoak GE, Moran C and Coffin JM: Role of endogenous retroviruses as mutagens: the hairless mutation of mice. Cell 54: 383-391, 1988.

17. Seperack PK, Mercer JA, Strobel MC, Copeland NG and Jenkins NA: Retroviral sequences located within an intron of the dilute gene alter dilute expression in a tissue-specific manner. EMBO J 14: 2326-2332, 1995.

18. Druker R and Whitelaw E: Retrotransposon-derived elements in the mammalian genome: a potential source of disease. J Inherit Metab Dis 27: 319-330, 2004.

19. Jun K, Lee SB and Shin HS: Insertion of a retroviral solo long terminal repeat in mdr-3 locus disrupts mRNA splicing in mice. Mamm Genome 11: 843-848, 2000.

20. Sun $\mathrm{H}$ and Chasin LA: Multiple splicing defects in an intronic false exon. Mol Cell Biol 20: 6414-6425, 2000.

21. Kol G, Lev-Maor G and Ast G: Human-mouse comparative analysis reveals that branch-site plasticity contributes to splicing regulation. Hum Mol Genet, 2005.
22. Lam BJ and Hertel KJ: A general role for splicing enhancers in exon definition. RNA 8: 1233-1241, 2002.

23. Wang Z, Rolish ME, Yeo G, Tung V, Mawson M and Burge CB: Systematic identification and analysis of exonic splicing silencers. Cell 119: 831-845, 2004

24. Cartegni L, Chew SL and Krainer AR: Listening to silence and understanding nonsense: exonic mutations that affect splicing. Nat Rev Genet 3: 285-298, 2002.

25. Blencowe BJ: Exonic splicing enhancers: mechanism of action, diversity and role in human genetic diseases. Trends Biochem Sci 25: 106-110, 2000.

26. Bailey JA, Liu G and Eichler EE: An Alu transposition model for the origin and expansion of human segmental duplications. Am J Hum Genet 73: 823-834, 2003.

27. Kang YK, Park JS, Lee CS, Yeom YI, Chung AS and Lee KK: Efficient integration of short interspersed element-flanked foreign DNA via homologous recombination. J Biol Chem 274: 36585-36591, 1999.

28. Cantrell MA, Filanoski BJ, Ingermann AR, et al: An ancient retrovirus-like element contains hot spots for SINE insertion. Genetics 158: 769-777, 2001.

29. Wilkinson MF: A new function for nonsense-mediated mRNAdecay factors. Trends Genet 21: 143-148, 2005.

30. Zhu H, Ryan K and Chen S: Cloning of novel splice variants of mouse mGluR1. Brain Res Mol Brain Res 73: 93-103, 1999.

31. Selkirk JV, Challiss RA, Rhodes A and McIlhinney RA: Characterization of an N-terminal secreted domain of the type- 1 human metabotropic glutamate receptor produced by a mammalian cell line. J Neurochem 80: 346-353, 2002.

32. Mason DJ: Glutamate signalling and its potential application to tissue engineering of bone. Eur Cell Mater 7: 12-26, 2004.

33. Coesmans M, Smitt PA, Linden DJ, et al: Mechanisms underlying cerebellar motor deficits due to mGluR1-autoantibodies. Ann Neurol 53: 325-336, 2003.

34. Serra HG, Byam CE, Lande JD, Tousey SK, Zoghbi HY and Orr HT: Gene profiling links SCA1 pathophysiology to glutamate signaling in Purkinje cells of transgenic mice. Hum Mol Genet 13: $2535-2543,2004$. 